Research Article

\title{
Personalized Recommendation for Mobile Internet Wealth Management Based on User Behavior Data Analysis
}

\author{
Xiangyu Ye ${ }^{1}$ and Mengmeng Chen $\mathbb{D}^{2}$ \\ ${ }^{1}$ DeHeng Law Offices (Wenzhou), Wenzhou, Zhejiang 325000, China \\ ${ }^{2}$ Wenzhou University of Technology, Wenzhou, Zhejiang 325000, China \\ Correspondence should be addressed to Mengmeng Chen; chenmengmeng@wzu.edu.cn
}

Received 17 September 2021; Revised 27 October 2021; Accepted 2 November 2021; Published 19 November 2021

Academic Editor: Rahman Ali

Copyright (c) 2021 Xiangyu Ye and Mengmeng Chen. This is an open access article distributed under the Creative Commons Attribution License, which permits unrestricted use, distribution, and reproduction in any medium, provided the original work is properly cited.

\begin{abstract}
Economic development has provided good opportunities for the development of securities companies. Similarly, the development of Internet technology has also brought huge opportunities and challenges to the development of securities companies. Aiming at the current wealth management issues in the era of mobile Internet, this article attempts to develop a personalized recommendation approach on the basis of users' behavioral data analysis. We analyzed and judged the current situation of mobile Internet wealth management using personalized recommendation systems. On the basis of personalized recommendation, we use the user's interest tags, personalized recommendation technology, and data mining technology to analyze and summarize customer transaction records. This is done through the use of preservation of customer transaction data. By understanding customers' investment needs, risk preferences, and other information, we can segment customers and provide them with targeted products and services. As a result of the study, a flexible personalized recommendation framework is designed and validated for mobile Internet wealth management services. The effectiveness of the proposed approach is verified through testing of the developed model.
\end{abstract}

\section{Introduction}

Traditional third-party wealth management is a provider of "financial products" and "professional services," acquiring customers through offline activities, development channels, and telemarketing [1]. In this way, material resources must be invested offline, and the cost is high. Mobile Internet wealth management companies guide users' attention through personalized and professional contents and accurately analyze customer preferences in the background, which in turn enables financial planners to provide peer-topeer services. This improves efficiency and saves costs $[2,3]$.

With the rapid development of mobile communication technology and mobile terminal equipment, the Internet has quickly entered the mobile Internet era. It solves the shortcomings of the Internet in time and space $[4,5]$. Users can use mobile terminal devices anytime and anywhere to surf the Internet. At the same time, technologies based on social networking and big data have progressed in the era of mobile Internet. This technology plays a significant role in the transformation of traditional industries [6]. This also puts forward higher requirements for third-party wealth management companies. How to attract users to do a good job in mobile Internet and wealth management at the same time is an important issue. The current mobile Internet wealth management model mainly displays products online, provides consulting services, and signs offline. This has changed the multilayer agency model of traditional thirdparty wealth management companies, compressed and eliminated channel vendors, and reduced transaction costs $[7,8]$. Claudia et al. concluded, by studying the innovation of the financial industry, that the rapid development of Internet technology has significant impact on the brokerage services [9]. Coor et al. conducted a study on Internet finance in the United States and found that Internet finance formed an industrial hierarchy and a complete industrial chain in 2000 
and completely subverted the operation of the traditional financial industry [10]. Yong et al. believe that it is possible to analyze high-value customers through data technology under the background of the Internet, adopt differentiated services for customers, and realize the realization of brokerage data resources, but it has gone through a relatively long process [11]. Guignard et al.'s research found that the product of the combination of Internet technology and securities firms will inevitably bring changes to the operating mode of securities firms. The most common way is the financial asset management of securities firms, especially when securities companies make investment portfolio decisions. Internet big data analysis must be used [12]. Under normal circumstances, the recommendation process of a personalized recommendation system is carried out in stages. The first is the interest modeling stage, the second is the item matching stage, and the last is the recommendation result feedback stage.

At present, almost all brokerages can realize online transactions. The digitization of offline on-site transactions has become a consensus among brokers. Automated services and intelligent services have become a trend in future brokerages' customer service management. On this basis, we found that the advantages and disadvantages of the two are complementary. Aiming at the current wealth management issues, this article attempts to develop a personalized recommendation scheme based on user behavior data analysis. We combine user interest tags and personalized recommendation technology and summarize customer transaction records through the storage of customer transaction data for information technology. Finally, we discuss the limitations of this article and future research prospects.

In this study, a personalized recommendation system for mobile Internet wealth management is proposed which is based on the user behavior data analysis. Key contributions of the study include the following:

(i) A flexible recommendation framework is proposed for mobile Internet wealth management services.

(ii) A comprehensive user profiling mechanism is developed which exploits the users' behavioral data.

(iii) On the basis of understanding the customer's investment needs and risk appetite and other information, we segment the customers and provide customized and personalized products.

\section{Related Technology Overview}

2.1. Wealth Management. In the business model of traditional brokerage firms, they mainly provide customers with business channels and charge a certain fee. Even under this model, the homogenization competition among brokerage firms is quite fierce $[13,14]$. However, the platform-based business network model has brought a huge impact to traditional brokers. Large-scale network platforms rely on their own resource integration advantages, not only do not need the profits of the brokerage industry, but take the way of subsidies to grab customers. The platform provides free channel services and even gives appropriate subsidies to selected customers. The platform subverts the traditional brokerage business model $[15,16]$. This new model has increased the stickiness of customers and established a new financial ecosystem to make up for the loss of the platform in terms of transaction commissions through customer consumption in other areas. Under the new Internet background, brokerage firms will not only face competition from their peers but also be challenged by other cross-industry competitors in the financial ecosystem [17]. Figure 1 reflects the schematic diagram of the mobile Internet wealth management system architecture.

Finance refers to financing of funds, and financial institutions are the media of financing [18]. The medium of financing that we are familiar with is the bank. The bank acts as a third party to connect the demanders of funds and the providers of funds to realize the allocation of resources [19]. With the establishment of online payment platforms, the intermediary role of traditional financial institutions is no longer important. Internet finance has emerged since then. Customers do not need to finance through banks or capital markets but can use Internet financial services and realize their own financial needs [20]. Subsequently, with the popularization of smart phones, Internet finance has extended to mobile clients, resulting in the birth of mobile Internet finance.

2.2. Personalized Recommendation. Generally, recommendation systems can broadly be divided into content-based, collaborative filtering, knowledge-based, and hybrid recommendation systems [21, 22]. Apart from these categories, the classification may include some other scattered classification methods, such as semantic-based, context-based, and grammar-based filtering methods [23]. The same methods can equally likely be used and applied to personalized recommendation systems in diverse fields of applications. At present, personalized recommendation systems are mainly divided into two categories according to different recommendation technologies: rule-based recommendation systems and information filtering systems. In addition, information filtering systems can be further divided into content-based filtering systems and collaborative filtering systems.

Under normal circumstances, the recommendation process of a personalized recommendation system is carried out in stages. The first is the interest modeling stage, the second is the item matching stage, and the last is the recommendation result feedback stage [24]. User interest modeling is mainly to obtain user interests and user needs and then generate an interest model that includes user preferences, background, and needs. Therefore, building an accurate user interest model is the basis for personalized recommendation systems for personalized recommendations. We then determine the recommended service effect $[25,26]$. The algorithm measures the correlation between users and association rules by comparing the attributes of users belonging to the same association rule. Economic development has provided good opportunities for the development of securities companies, and the development of 


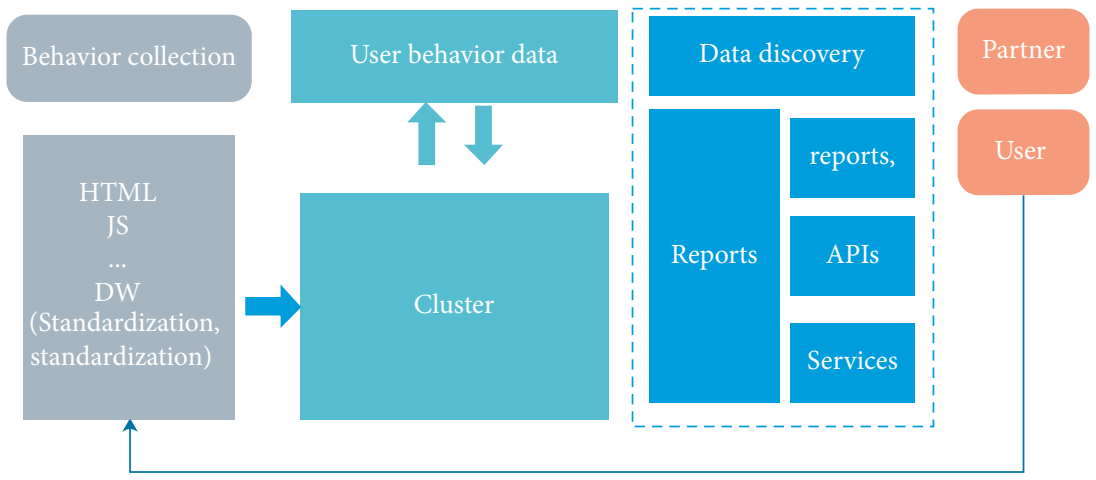

Figure 1: Schematic diagram of the mobile Internet wealth management system architecture.

Internet technology has also brought huge opportunities for the development of securities companies. If the association rules are correctly evaluated, the accuracy of the filtering algorithm needs to be further confirmed.

In the second phase of item matching, filtering techniques and algorithms are used. These may include collaborative filtering algorithms and vector matching algorithms. Standard collaborative filtering algorithms are based on data statistics while vector matching algorithms are deep-level models based on machine learning. For example, alternating least squares (ALS) method is a typical matrix factorization method which generates a user embedding table and item embedding table based on behavior data tables [27].

In the third stage of recommendation and user feedback, the recommendation system prompts the user via system interfaces to provide feedback using the ratings for items. This way, the constructed personalized recommendation models are improved. The accuracy of recommendation models is measured using quantitative ratings of the user. The feedback can be implicit, explicit, or hybrid depending on the type of the recommendation system [23].

Zhang et al. proposed a method of merging multiple user profiles in a context-based environment to resolve conflicts [28]. Li et al. introduced a Bayesian network-based method, which is effective and scalable for data stream learning and provides users with personalized services [29].

\section{Mobile Internet Wealth Management Based on Personalized Recommendation}

3.1. Internet User Interest Tags. Data features mainly refer to data related to the user's interests, features, and preferences. The extraction of these data is the process of model data preparation, mainly for the establishment of the user's interest model. Generally speaking, the extraction of data features can be divided into two parts: explicit information (explicit data) extraction and implicit information (implicit data) extraction. Figure 2 shows the proofreading process of the interest knowledge graph.

The extraction of explicit information requires users to actively provide data that can express their interests, such as the user's rating of a certain item, a clear statement of a certain item, and the user's personal information. The process of extracting explicit information is simple and direct, which helps to speed up the model formation, but it also requires users to spend a certain amount of time and energy to participate in the process. The user interest model is one of the key parts of the personalized recommendation system. The quality of the model is directly related to the recommendation quality of the recommendation system. In fact, the interest model is a data structure, and its form of expression directly determines the user model's ability to reflect user interests and the model's computability.

\subsection{Recommendations Based on Personalized Wealth} Management. The algorithm is based on the basic idea that similar users have similar interests and hobbies. It is the earliest and most successful recommendation application technology. This section proposes the collaborative filtering recommendation algorithm $\mathrm{M}-\mathrm{CF}$ based on the user interest model and uses the real-time user interest model proposed in Section 4 to express the user's hobbies. Economic development has provided good opportunities for the development of securities companies, and the development of Internet technology has also brought huge opportunities for the development of securities companies. The real-time user interest model can obtain the user's hobby according to the user's browsing interest, which can be effective; at the same time, the number of standard tags is much smaller than the number of items. According to the user-standard tag matrix, the similarity between users can be directly calculated. There is no need to consider the problem of filling values. In addition, the algorithm does not need to actively provide items. The scoring data effectively reduce the degree of cooperation between the system and user participation. The flowchart of the personalized recommendation system is shown in Figure 3.

The selection of neighbors is to establish a neighboring user group for the target user, placing users with similar interests and hobbies in the neighboring user group, and using neighboring users' evaluations of the project to provide recommendations to the target user. The selection process of neighbor users is based on the user-item rating matrix as the data source, the similarity between users is calculated, and $K$ users with higher similarity are selected as neighbors. 


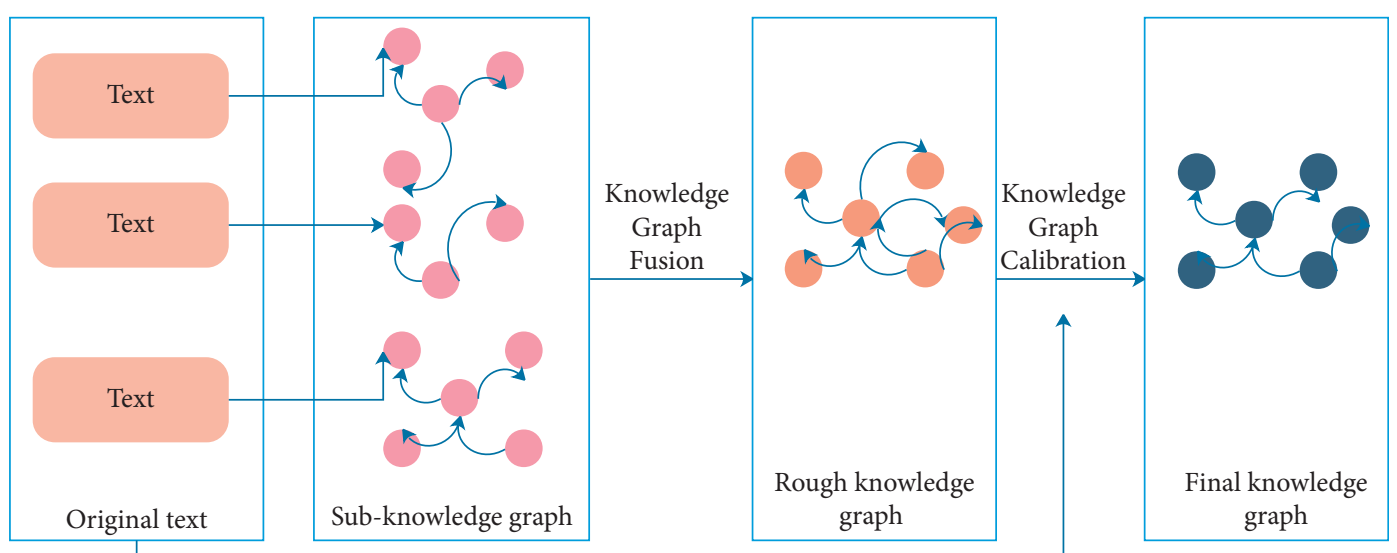

FIGURE 2: Proofreading process of interest knowledge graph.

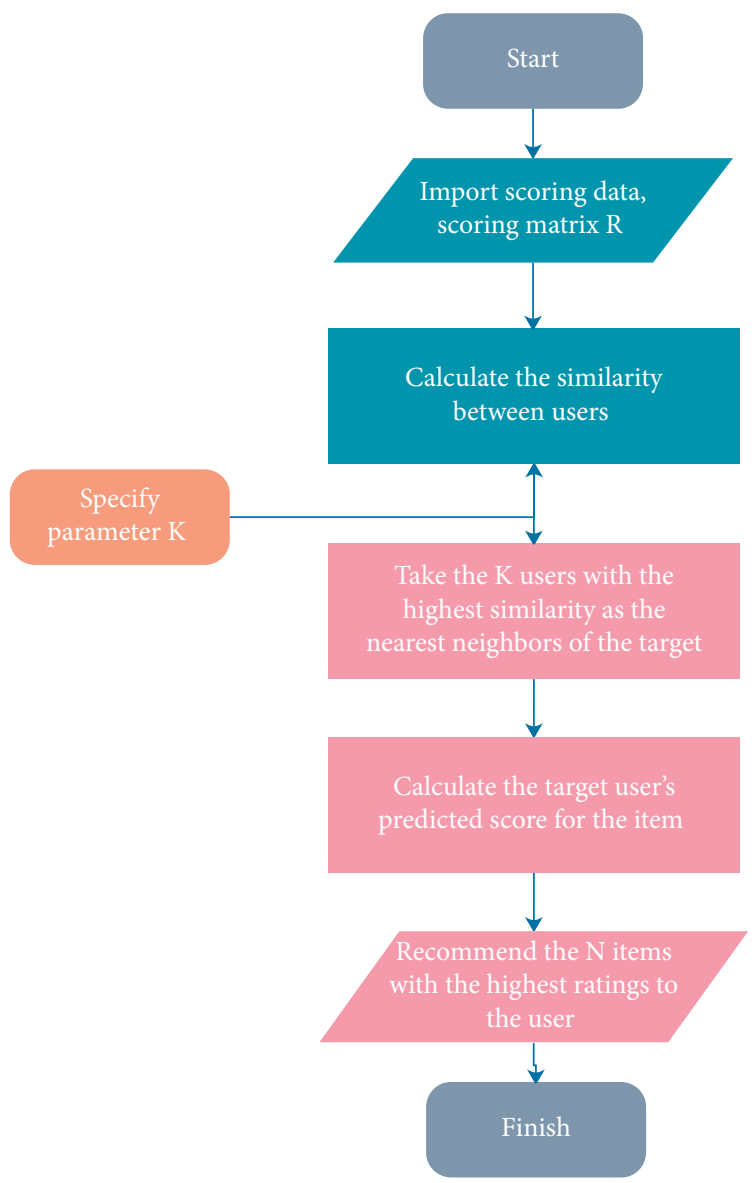

FIgURE 3: Flowchart of personalized recommendation system.

There are many ways to calculate similarity between users. Commonly used methods include cosine similarity.

$$
\delta_{k}=o_{k}\left(t_{k}-o_{k}\right)=o_{k}\left(1-o_{k}\right)\left(t_{k}-o_{k}\right) .
$$

Equation (1) is used to determine the similarity level between the contents for retrieving relevant ones in which the user is interested.

After getting the user's neighbors, the scores of other neighboring users can be used to predict the scores of items that the target user has not evaluated and then make recommendations to the target users.

$$
\delta_{n}=\left(O_{n}-T_{n}\right) O_{n}\left(1-O_{n}\right) .
$$

Equation (2) is used to determine relevancy scores between the contents.

Generally, there are two types of results for the target user's recommendation. One is the user's rating prediction for any item, and the other is Top-N recommendation. 
However, since the experiment only involves the verification of the accuracy of the user's interest items and does not involve the verification of the weight of the interest item and the model update algorithm, the verification of this part will be reflected in the experiments described in this section, and the experiments described in this section also recommend improvements. The accuracy, precision, and response time of the algorithm are further verified.

$$
\begin{aligned}
& P(y=1 \mid t ; \theta)=h_{\theta}(t), \\
& P(y=0 \mid t ; \theta)=1-h_{\theta}(t) .
\end{aligned}
$$

That is, we calculate the user's predicted scores for all unevaluated items and select the Top- $\mathrm{N}$ items with the highest predicted scores and recommend them to the user.

Equation (3) is used to select Top- $\mathrm{N}$ items based on the relevancy score.

$$
\begin{aligned}
Z_{2} & =\max \sum_{k=1}^{n} d\left(S_{k}\right), \\
B^{(x)} & =A \cdot R^{(x)}=\left(b_{1}^{(x)}, b_{2}^{(x)}, b_{3}^{(x)}, b_{4}^{(x)}, b_{5}^{(x)}\right) .
\end{aligned}
$$

The weighted synthesis module is mainly set up to deal with the different situations faced by the recommendation system at different times, so that the collaborative filtering algorithm in the early cold start of the recommendation system can be alleviated. When the data are abundant in the later period, the user's new potential interest cannot be found.

$$
k_{i}^{q}=\sqrt{\sum_{j}\left(\omega_{1 j i}-x_{j}^{q}\right)} \times b_{1 i} .
$$

Therefore, it is necessary to adjust the contribution of each algorithm in different periods through the setting of weights to achieve the purpose of maximizing strengths and avoiding weaknesses.

$$
f 1(x)=\frac{1}{1+e^{-A x}} .
$$

User data in different environments have their own characteristics, so the method for determining the weight of this system is to divide the data into a test set and a training set.

$$
\Delta w_{j i}=\eta \delta_{j} x_{j i}
$$

During the model establishment, the personalization algorithm is trained multiple times through the training set data, and different values of $a$ and $p$ are taken each time, so as to obtain the weights $a$ and $p$ with the best performance.

$$
a=\frac{\sum\left(y_{i} / \sigma_{i}^{2}\right) e^{b\left(x_{1 i}+x_{2 i}\right)}}{\sum\left(1 / \sigma_{i}^{2}\right) e^{2 b\left(x_{1 i}+x_{2 i}\right)}}
$$

Under normal circumstances, the recommendation process of a personalized recommendation system is carried out in stages. The first is the interest modeling stage, the second is the item matching stage, and the last is the recommendation result feedback stage.

\section{Personalized Recommendation Model Test}

User interest model and recommendation algorithm are the two cores of personalized recommendation. This section focuses on the main line of personalized recommendation. In Section 3, the method of establishing real-time performance through tags is adopted, and the model is verified through experiments. However, since the experiment only involves the verification of the accuracy of the user's interest items and does not involve the verification of the weight of the interest item and the model update algorithm, the verification of this part will be reflected in the experiments described in this section, and the experiments described in this section also recommend improvements. The accuracy, precision, and response time of the algorithm are further verified. This new model has increased the stickiness of customers and established a new financial ecosystem to make up for the loss of the platform in terms of transaction commissions through customer consumption in other areas. Under the new Internet background, brokerage firms will not only face competition from their peers but also be challenged by other cross-industry competitors in the financial ecosystem.

4.1. Data Source. This article selects a well-known mobile Internet wealth management company as the data source. China Mobile Ltd, which is the world's biggest wireless operator by subscriber numbers and which has improved its inactive performance by actively promoting its mobile Internet business, is used for collecting the data. The data contain a large amount of user information and user data, which can provide richer data support for the subject research of this article. The data contain profile data, mobile data usage information, their interaction on the web and tags, etc. It is evident from the relevant research that tags and film reviews used by users reflect the users' cognitive characteristics. Similarly, it also shows the resource descriptions when users add tags, make comments, and share their perceptions of the content or form of resources. Therefore, this article selects tags and wealth preferences.

Experiments are conducted to identify the basic data of the wisdom and cognition of the user group.

4.2. Model Testing. In order to optimize the performance of the personalized recommendation system, it is necessary to determine the respective weights of collaborative filtering and content-based filtering, that is, the size of $a$ and $p$. The values of $a$ and $p$ are divided into eleven stages to test the value of the personalized recommendation system in the test set under the $F$-measure indicator to determine the optimal combination weight. Then, we compare the personalized algorithm (PF), the user-based collaborative filtering algorithm $(\mathrm{CF})$, and the content-based filtering algorithm (CB) for the measurement ( $F$-measure). The four nearest neighbors of the recommendation results are evaluated separately. Figure 4 shows the comparison results of different recommendation algorithms when the nearest neighbor values are different. 


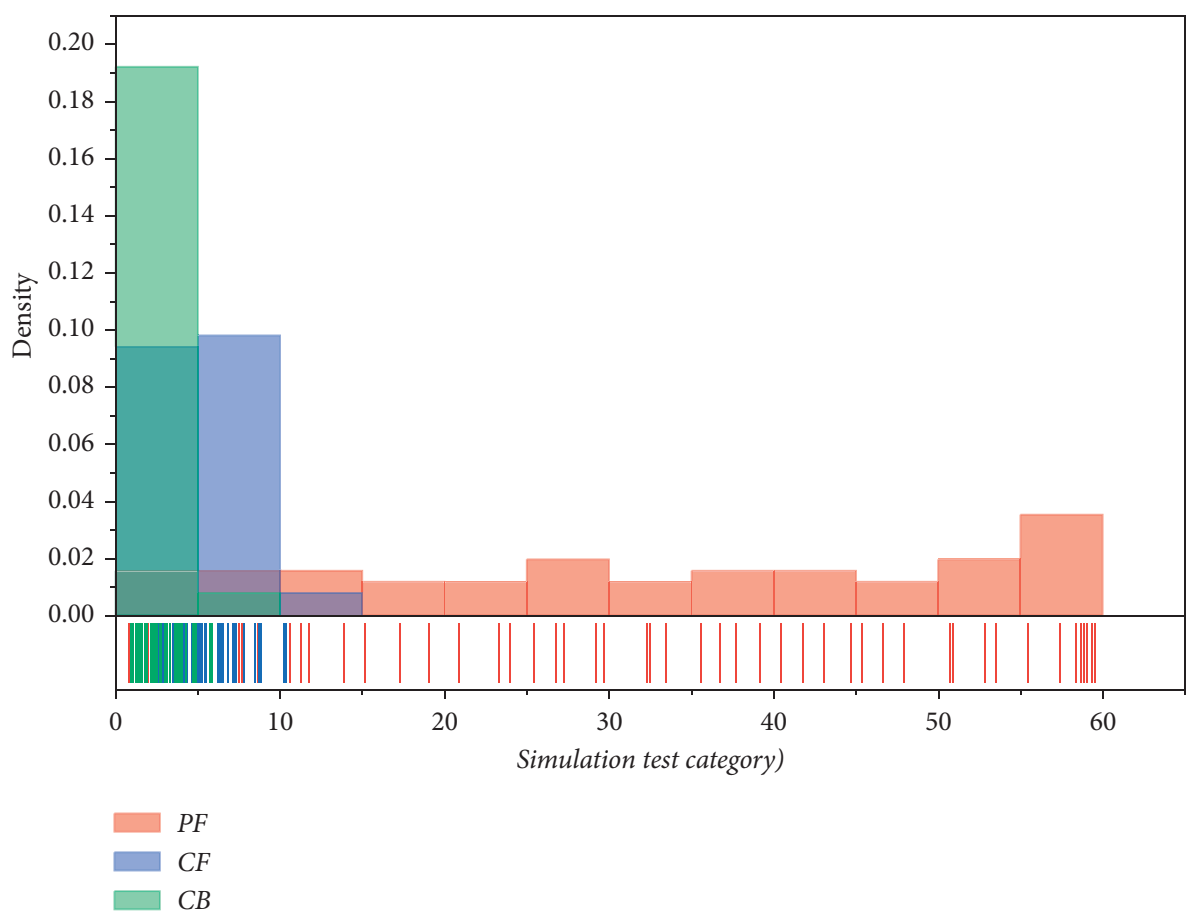

FIgURE 4: Comparison results of different recommendation algorithms when the nearest neighbor value is different.

It can be seen from Figure 4 that the accuracy of the recommendation algorithm in this paper is higher than that of the traditional recommendation algorithm. The average difference of item scores is measured by the improved average difference formula of scores, even if the lower correlation is mixed. Users can also reduce the contributions of users with low similarity to improve the accuracy of recommendation through the improved item score difference formula. It can also be seen from the graph that the number of nearest neighbors as the recommendation result is not better. The three algorithms perform best when the nearest neighbor is 6 at the same time.

With the increase in the number of neighbor users, the intersection of the item set of neighbor users and the items to be predicted in the test set gradually becomes larger. At present, almost all brokerages can realize online transactions. The onlineization of offline on-site transactions has become a consensus among brokers.

For the experimental setup, when the nearest neighbor values are different, the significance of the result of the proposed (PF) personalized recommendation method can be calculated using the degrees of freedom and variance value. The $p$ values are checked and compared to the significance level or rather, the alpha. A $p$ value less than 0.05 shows that the obtained results are statistically significant.

Figure 5 shows the impact of different user interest models on recall.

A user interest model is a recommendation model which describes a user's interests exactly in order to improve the quality of information access and accurate recommendations. These types of models are challenging from different perspectives, such as initial user information acquisition, user profile representation, and description of user interest evolution [30].

It can be seen from Figure 5 that the overall recall rate of the $\mathrm{CF}$ algorithm and the M-CF algorithm is not very high, and the recommendation threshold can be adjusted to improve the recall rate. When the value of the number of neighbor user's $K$ is about 35, the recall rate of the CF algorithm and the $\mathrm{M}-\mathrm{CF}$ algorithm is equivalent. When $K>35$, the recall rate of the $\mathrm{M}-\mathrm{CF}$ algorithm is higher. Analysis of the reasons found that when there are few neighbor users, the intersection of the neighbor users' item set and the test set to be predicted scoring items is small, resulting in a large deviation between the predicted scores of some items and the actual scores, so the average absolute error is higher and the recommendation accuracy is relatively high. Experiments show that the user interest model based on standard tags can improve the recommendation quality of the algorithm to a certain extent. The main innovation of this chapter is to use the user's interest model to calculate the similarity between users and establish an approximate user group of target users, which narrows the search scope of related items and improves the credibility of item ratings. Automated services and intelligent services have become a trend in future brokerages' customer service management. On this basis, we found that the advantages and disadvantages of the two are complementary. The algorithm proposes an improved item score difference formula based on user similarity to increase the contribution of similar users in the item score difference. Experiments show that this improvement improves the accuracy of algorithm recommendation. 


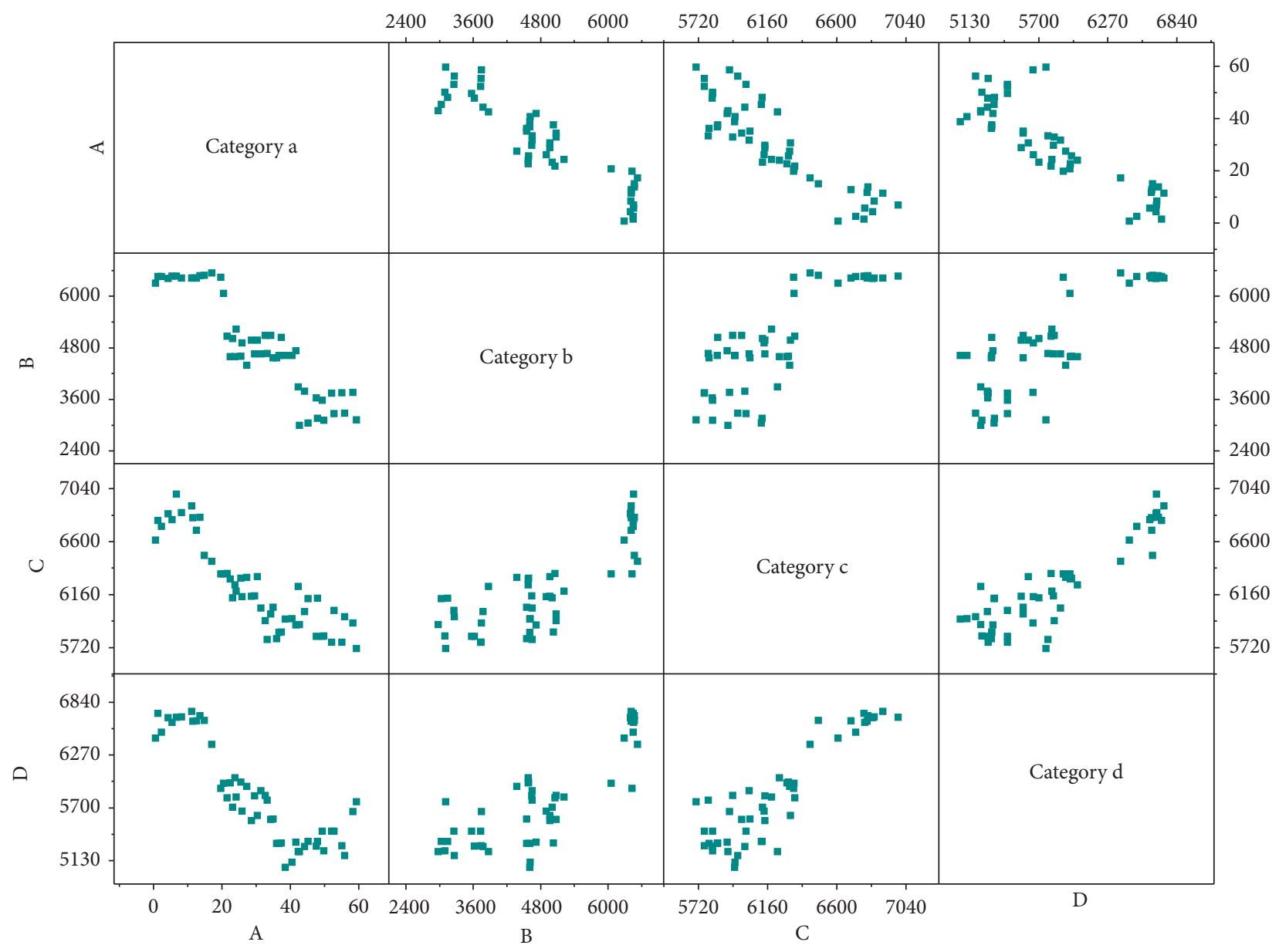

FIgURE 5: The impact of different user interest models on recall.

\section{Conclusion}

Economic development has provided good opportunities for the development of securities companies, and the development of Internet technology has also brought huge opportunities for the development of securities companies. At present, almost all brokerages can realize online transactions. Under the new Internet background, brokerage firms will not only face competition from their peers but also be challenged by other cross-industry competitors in the financial ecosystem. The onlineization of offline onsite transactions has become a consensus among brokers. Automated services and intelligent services have become a trend in future brokerages' customer service management. On this basis, we found that the advantages and disadvantages of the two are complementary. For example, the content-based filtering algorithm does not have the cold motion problem of the collaborative filtering algorithm. Algorithms based on content filtering are difficult to process non-text objects such as sound and video. The personalized recommendation algorithm based on weighted integration of content uses test set data to train the model to obtain a weighted comprehensive weight.
Small and medium-sized securities firms have made efforts in service innovation and have made a lot of efforts in exploring new product development and new profit models. Whether it is a medium-to-large brokerage firm or a small brokerage firm, big data analysis and cloud computing platforms must become the basis for their development. Customer-centric service orientation will guide them to work around customer needs.

In future research, we are plaining to work more specifically on the particular type of recommendation methods. Actually, the personalization is more effectively managed using content-based and knowledge-based approaches. Hence, in future we plan to conduct an in-depth study in these directions rather than going general.

\section{Data Availability}

The data used to support the findings of this study are included within the article.

\section{Conflicts of Interest}

The authors declare that they have no conflicts of interest. 


\section{References}

[1] H. Geng, M. Cheng, and J. Zhang, "Effects of wealth management products on bank risk in China: the role of audit committee effectiveness," Pacific Economic Review, vol. 13, no. 10, pp. 332-345, 2021.

[2] M. D. A and J. G. S. B, "A note on a dynamic goal-based wealth management problem," Finance Research Letters, vol. 4, no. 2, pp. 231-243, 2021.

[3] S. R. Dasa, D. Ostrova, A. Radhakrishnanb, and D. Srivastav, "Dynamic optimization for multi-goals wealth management," Journal of Banking \& Finance, vol. 16, no. 9, pp. 371-386, 2021.

[4] J. C. Sharman, The Despot's Guide to Wealth Management: On the International Campaign against Grand Corruption, Cornell University Press, Ithaca, NY, USA, 2017.

[5] L. Scott and S. Cavaglia, "A wealth management perspective on factor premia and the value of downside protection," Journal of Portfolio Management, vol. 43, no. 3, pp. 33-41, 2018.

[6] J. J. Mangano, "Wealth management unwrapped: unwrap what you need to know and enjoy the present," Financial Analysts Journal, vol. 71, no. 5, pp. 76-77, 2015.

[7] B. R. Munier, "On bespoke decision-aid under risk: the engineering behind preference elicitation," IMA Journal of Management Mathematics, vol. 29, no. 3, pp. 253-273, 2016.

[8] R. Nanni, "The "China" question in mobile internet standardmaking: insights from expert interviews," Telecommunications Policy, vol. 45, 2021.

[9] B. Claudia, K. Fanny, S. Filip, and E. D. Daniel, "A systematic review of trial-based economic evaluations of internet- and mobile-based interventions for substance use disorders," The European Journal of Public Health, vol. 31, no. 31 Suppl 1, pp. i19-i28, 2019.

[10] A. Coor, R. Kachur, A. Friedman et al., "Sexually transmitted disease, human immunodeficiency virus, and pregnancy testing behaviors among internet and mobile dating application users and nonusers, 2016," Sexually Transmitted Diseases, vol. 46, no. 8, pp. e83-e85, 2019.

[11] P. Yong, W. Zhao, M. Q. Cao, L. Du, and J. C. Chen, "The effect of remote health intervention based on internet or mobile communication network on hypertension patients: protocol for a systematic review and meta-analysis of randomized controlled trials," Medicine, vol. 98, no. 9, Article ID e14707, 2019.

[12] J. Guignard, P. Senn, R. Koller, M. Caversaccio, M. Kompis, and G Mantokoudis, "Mobile internet telephony improves speech intelligibility and quality for cochlear implant recipients," Otology \& NeurotologyOfficial Publication of the American Otological Society, American Neurotology Society [and] European Academy of Otology and Neurotology, vol. 40, no. 3, p. e206, 2019.

[13] Z. Wang, L. Gao, T. Wang, and J. Luo, "Monetizing edge service in mobile internet ecosystem," IEEE Transactions on Mobile Computing, vol. 7, no. 3, pp. 78-90, 2020.

[14] S. Gupta, E. M. Johnson, J. G. Peacock et al., "Radiology, mobile devices, and internet of things (iot)," Journal of Digital Imaging, vol. 33, no. 12, pp. 735-746, 2020.

[15] Z. Ning, J. Huang, X. Wang, J. Rodrigues, and L. Guo, "Mobile edge computing-enabled internet of vehicles: toward energyefficient scheduling," IEEE Network, vol. 33, no. 5, pp. 1-8, 2019.

[16] L. Ma, X. Wang, L. Wang, and M. Huang, "Tcda: truthful combinatorial double auctions for mobile edge computing in industrial internet of things," IEEE Transactions on Mobile Computing, vol. 11, no. 6, pp. 164-175, 2021.

[17] P. Zhang, M. Durresi, and A. Durresi, "Internet network location privacy protection with multi-access edge computing," Computing, vol. 1376, pp. 1-18, 2020.

[18] H. Y. Jeong, "Multi criteria based personalized recommendation service using analytical hierarchy process for airbnb," The Journal of Supercomputing, vol. 77, pp. 1-19, 2021.

[19] Y. Chen, Y. Dai, X. Han, Y. Ge, H. Yin, and P. Li, "Dig users' intentions via attention flow network for personalized recommendation," Information Sciences, vol. 547, pp. 1122-1135, 2021.

[20] B. Hui, L. Zhang, X. Zhou, X. Wen, and Y. Nian, "Personalized recommendation system based on knowledge embedding and historical behavior," Applied Intelligence, vol. 8, no. 11, pp. 427-443, 2021.

[21] C. Zeng, C. X. Xing, and L. Z. Zhou, "A survey of personalization technology," Journal of Software, vol. 13, no. 10, pp. 1952-1961, 2002.

[22] M. Afzal, S. I. Ali, R. Ali et al., "Personalization of wellness recommendations using contextual interpretation," Expert Systems with Applications, vol. 96, pp. 506-521, 2018.

[23] F. O. Isinkaye, Y. O. Folajimi, and B. A. Ojokoh, "Recommendation systems: principles, methods and evaluation," Egyptian informatics journal, vol. 16, no. 3, pp. 261-273, 2015.

[24] Q. A. Tian, W. A. Chi, A. Gc, and B. Lxz, "Temporal information effect on personalized recommendation - sciencedirect," Chinese Journal of Physics, vol. 62, pp. 213-222, 2019.

[25] J. Shen, Z. Cheng, M. Yang, B. Han, and S. Li, "Style-oriented personalized landmark recommendation," IEEE Transactions on Industrial Electronics, vol. 66, pp. 9768-9776, 2019.

[26] W. Wu, R. Zhang, and L. Liu, “A personalized network-based recommendation approach via distinguishing user's preference," International Journal of Modern Physics B, vol. 33, no. 06, Article ID 1950029, 2019.

[27] D. Das, L. Sahoo, and S. Datta, "A survey on recommendation system," International Journal of Computer Applications, vol. 160, no. 7, pp. 6-10, 2017.

[28] C. Zhang, T. Li, Z. Ren, Z. Hu, and Y. Ji, "Taxonomy-aware collaborative denoising autoencoder for personalized recommendation," Applied Intelligence, vol. 49, no. 3, 2019.

[29] G. Li, J. Zhuo, C. Li, J. Hua, and H. Zhang, "Multi-modal visual adversarial bayesian personalized ranking model for recommendation," Information Sciences, vol. 572, no. 1, 2021.

[30] H. Li, Y. Shen, C. Wang, H. Yang, and R. Ali, "Reading behaviour based user interests model and its application in recommender system," in Proceedings of the 2017 IEEE 2nd Advanced Information Technology, Electronic and Automation Control Conference (IAEAC), pp. 683-690, IEEE, Chongqing, China, March 2017. 